\title{
ACCIONES DE PREVENCIÓN EN ACOSO ESCOLAR UTILIZADAS POR DOCENTES DE EDUCACIÓN SECUNDARIA OBLIGATORIA
}

\author{
Amparo Pérez Carbonell \\ Genoveva Ramos Santana \\ Universitat de València \\ Macarena Serrano Sobrino \\ Universidad Internacional de La Rioja
}

\begin{abstract}
RESUMEN: El objetivo de este artículo es presentar la revisión de distintas investigaciones que permiten conocer las acciones de prevención primaria, secundaria y terciaria que los docentes de Educación Secundaria Obligatoria (ESO) realizan ante el acoso escolar o bullying. Actualmente los distintos agentes educativos y sociales muestran una gran preocupación por las crecientes denuncias del alumnado de esta etapa educativa. A tal fin, se realiza un proceso sistemático de indagación documental en distintas bases de datos sobre estudios, en el contexto educativo español, realizados en torno a esta temática. Se concluye, por un lado, que el profesorado encuestado, conoce y desarrolla más acciones de prevención primaria y secundaria sobre el acoso escolar que de prevención terciaria. Que las acciones primarias y secundarias tienen un enfoque más educativo que punitivo o sancionador y buscan principalmente la generación de un clima adecuado de convivencia tanto en el centro como en el grupo-clase. Por otro lado, en el nivel de prevención terciaria, desarrollan acciones centradas en hablar con el alumnado, aplicar las normas de disciplina y convivencia y derivar los casos a los departamentos de orientación de sus centros.
\end{abstract}

PALABRAS CLAVE: Sistema educativo, acoso, enseñanza secundaria, formación de docentes de secundaria, joven.

\section{PREVENTION ACTIONS IN SCHOOL BULLYING USED BY TEACHERS OF SECONDARY EDUCATION}

\footnotetext{
ABSTRACT: The aim of this paper is to present a review of various studies that provide insight into the actions of primary, secondary and tertiary prevention teachers of Secondary Education (ESO) against school bullying or harassment.
} 
Currently the various educational and social agents show great concern for the increasing reports of students in this educational stage. To this end, this project completed a systematic process of documentary research in different databases on studies pertaining to this subject in the Spanish educational context. It was concluded, firstly, that teachers surveyed know and develop more primary and secondary prevention actions against bullying than tertiary prevention actions. Primary and secondary actions have a more educational than punitive or disciplinary approach and mainly seek to generate a climate of coexistence both in the center and in the class group. On the other hand, at the level of tertiary prevention, they develop actions focused on talking with students, applying the standards of discipline and coexistence and referring cases to the departments to target their centers.

KEYWORDS: Educational systems, bullying, Secondary education, secondary teacher education, young.

Recibido: 2/12/2015

Aceptado: 16/06/2016

Correspondencia: Amparo Pérez Carbonell, Universitat de València, Departamento MIDE de la Facultad de Filosofía y CC. de la Educación, Avenida Blasco Ibáñez, 13, 46010, Valencia. Email: amparo.perez@uv.es.

\section{INTRODUCCIÓN}

Hablar de acoso escolar es hacerlo de una problemática que afecta principalmente al alumnado de Institutos de Educación Secundaria (IES). Esta se define como una "conducta agresiva, intencional y mantenida en el tiempo, perpetrada por un individuo o grupo de ellos contra otro, al que consideran su víctima habitual" (Cerezo, 2012, p. 24).

Somos conscientes que en los últimos años se han realizado numerosos estudios entorno a la problemática del acoso escolar, para conocer su nivel de incidencia, identificar a sus protagonistas o enumerar posibles factores de riesgo (Chaux, 2012; Defensor del pueblo, 2007; Del Barrio, 2013; García-Fernández, 2013; ISEI-IVEI, 2009; Ortega, 2010; Serrate, 2007). Sin embargo, son menores las investigaciones que se centran en describir el tipo de acciones que se siguen o que se deberían seguir para abordar educativamente este fenómeno en el ámbito escolar (Garaigordobil y Oñaderra, 2010; Valverde, 2013).

En España, hasta la publicación del Informe del Defensor del Pueblo sobre Violencia Escolar en 1999, y más concretamente hasta que sucede el trágico caso de Jokin Ceberio en el año 2004 -joven vasco de casi catorce años que se quita la vida a causa del acoso escolar que sufrió por parte de sus compañeros/as de clase- las investigaciones educativas no dirigen su mirada hacia esta problemática (Gabarda, 2015).

Marchesi, Pérez, Pérez y Marín (2007) en un estudio realizado en la Comunidad Valenciana señalan que para un $83,30 \%$ del profesorado, los casos de acoso escolar 
son muy o algo importantes para la consecución de la convivencia en los centros educativos; además, y en este mismo estudio, el alumnado de Educación Secundaria Obligatoria (ESO), destaca que dicho acoso hacía referencia sobre todo a hechos como insultar, poner motes y hablar mal de alguien.

En esta línea, un estudio estatal elaborado por Díaz-Aguado, Martínez y Martín en 2010 concluye que el acoso escolar es un fenómeno que perturba bastante o mucho la convivencia escolar, en opinión del profesorado, de los departamentos de orientación y de los equipos directivos. En este mismo estudio el alumnado destaca como hechos más frecuentes, hablar mal de alguien e ignorarle. Posteriormente y tras un proceso de investigación realizado por Ovejero, Smith y Yubero (2013) se constata que un $6 \%$ de los niños y niños/as y jóvenes españoles/as sufre acoso escolar.

Actualmente y debido a la elevada proporción de uso de las tecnologías de la comunicación entre los jóvenes, se dan fenómenos de acoso entre la población juvenil que también se producen en el mundo virtual, como es el caso del ciberbullying. Algunos autores consideran que el ciberbullying no es más que una continuidad del acoso escolar tradicional o bullying (Kovalski, Limber y Agaston, 2008). Otros autores, sin embargo (Jäger, Amado, Matos y Pessoa, 2010) afirman que las características únicamente propias del acoso a través de medios electrónicos como el anonimato del agresor/a y la posibilidad de traspasar los límites espacio temporales, hacen que encontremos dos fenómenos diferenciados. No obstante, la mayoría de los estudios señalan la existencia de una indiscutible relación entre el bullying y el ciberbullying reflejada en características como la existencia de desequilibrio de poder entre la víctima y su agresor/a, la durabilidad -comportamientos repetidos a lo largo del tiempoy la naturaleza agresiva de la conducta por parte del agresor/a, siendo el instrumento empleado lo que establece la diferencia clave entre ambos tipos de acoso (Gabarda, 2015). Por esto, diferentes estudios han encontrado solapamientos entre las formas tradicionales de acoso y las ejercidas a través de las tecnologías de la comunicación. De hecho, se afirma que existe una implicación entre, (en el 70-90\% dependiendo del estudio realizado), aquellos que dicen participar en el fenómeno del bullying tanto de manera tradicional como en el mundo virtual. Del Barrio (2013) establece que tomando el total de quienes se declaran cibervíctimas, la mayoría también son o han sido víctimas de otras formas de bullying (en el 89,5\% de los casos).

A nuestro modo de entender, es tarea de los centros educativos de Educación Secundaria Obligatoria (ESO) y de los agentes implicados en los mismos trabajar para la no violencia en todos los ámbitos de la vida personal, social y familiar de los/las estudiantes, y en especial en el del acoso escolar. Así, creemos imprescindible incluir en sus planes de convivencia un plan anti-bullying en el que se incluyan acciones orientadas a la sensibilización, formación, prevención e intervención en los casos de acoso entre iguales que integren a toda la comunidad educativa. Es decir, en los centros educativos se deben realizar acciones, que como bien señala Sag (2008) han de ser de prevención primaria, secundaria y terciaria.

Somos conscientes que la investigación sobre el bullying y los programas de intervención para prevenir o paliar sus efectos tienen una trayectoria de décadas de estudio (Ortega, Del Rey y Casas, 2013; Rigby y Smith 2011). En dicha trayectoria son reseñables las aportaciones que describen programas exitosos donde se han obtenido evidencias empíricas de la eficiencia de estrategias que abordan diferentes elementos 
del contexto escolar (Baldry y Farrington 2007; Olweus 2012; Pearce, Cross, Monks, Waters y Falconer, 2011; Ttofi y Farrington 2011). Por otro lado, estas investigaciones realizadas en los últimos años hacen patente la necesidad de romper la ley del silencio ante los distintos casos de acoso. En este sentido, Canals (2010) señala que las familias, el alumnado, el profesorado, los centros y el resto de la comunidad educativa son conocedores de las situaciones problema que ocurren en los propios centros educativos, sin embargo, por diversas razones, los hechos o casos de bullying no son tan conocidos como deberían serlo. Siguiendo a Garaigordobil (2011), en España es necesario realizar más estudios que permitan aportar datos más precisos, tanto para identificar la gravedad del bullying y el ciberbullying como para identificar parámetros relevantes para la prevención y la intervención de este fenómeno.

De todas las audiencias que pueden estar implicadas en los procesos de acoso escolar, el estudio que aquí presentamos se centra en el papel del profesorado (Del Barrio, 2007; Serrano e Iborra, 2005; Serrano, 2013), y de manera muy concreta, en el tipo de acciones que éste utiliza ante el acoso escolar. Con ello pretendemos que tanto el profesorado como las personas responsables de la gestión y dirección de los centros de ESO, sean conocedores de las estrategias de prevención primaria, secundaria y terciaria que se realizan y reflexionen sobre su adecuación, actualización y/o necesidad de cambio. Reflexión que puede llevar tanto al profesorado como a la dirección de un centro educativo al desarrollo de acciones o programas educativos de prevención e intervención sobre el acoso escolar de gran eficacia y eficiencia.

\section{Método}

Se realiza un estudio descriptivo-exploratorio de naturaleza cualitativa orientado a conocer cómo el profesorado de ESO actúa ante el acoso escolar en el contexto concreto de sus aulas y de su centro educativo. Así, las fases del proceso de indagación realizado fueron:

1. Proceso sistemático de revisión bibliográfica en distintas bases de datos como: Education Resources Information Center -ERIC-; Catálogo de artículos de revistas sobre ciencias sociales y humanidades -ISOC-; Red de Bases de Datos de Información Educativa del Ministerio de Educación y Ciencia -REDINED-; Catálogo de Investigaciones Educativas del Ministerio de Educación y Ciencia -CIDE-, entre otras.

2. Análisis documental-descriptivo de todos los estudios encontrados, atendiendo a los siguientes indicadores: a) Estudios y/o investigaciones en los cuales se analizan o desarrollan programas de prevención e intervención educativa entorno al acoso escolar o bullying en el contexto educativo español. b) Acciones de prevención e intervención educativa en el ámbito del acoso escolar o bullying en ESO. c) Acciones en el nivel de prevención e intervención que se desarrolla: primaria y/o secundaria y/o terciaria.

3. Planteamiento de resultados según la variable cronológica, el lugar de realización, el tipo de acciones y el porcentaje de profesorado que tras ser encuestado en los estudios analizados responde que realiza dichas acciones en su centro y/o aula. 


\section{Resultados}

Según los estudios revisados de forma sistemática, el profesorado de ESO ante el acoso escolar realiza distintas acciones de prevención: primaria, secundaria y terciaria -ver tablas de 1 a 3-. Siguiendo a Muñoz (2009): la prevención primaria, es aquella que hace el profesorado o el propio centro, de forma anticipada para evitar que aparezca esta problemática; la prevención secundaria, es aquella donde las actuaciones versan sobre la detección y control de los posibles factores de riesgo que puedan derivar en incipientes casos de bullying y, la prevención terciaria es la que incluye acciones dirigidas a corregir o emendar una situación de acoso escolar ya existente.

\section{Resultados para el nivel de prevención primaria y secundaria}

Las estrategias que se siguen en cada uno de estos niveles de prevención son diferentes. En la tabla 1, se presentan, en orden cronológico de ejecución del estudio y a modo de resumen, las acciones en aulas y/o centros que emplea el profesorado.

Tabla 1. Acciones de prevención primaria y secundaria que sigue el profesorado de ESO

\begin{tabular}{|c|c|c|}
\hline $\begin{array}{l}\text { Lugar de } \\
\text { realización }\end{array}$ & ESTUDIOS & ACCIONES PREVENCIÓN PRIMARIA Y SECUNDARIA** \\
\hline Cataluña & $\begin{array}{l}\text { Bisquerra y Martínez } \\
\text { (1998) }\end{array}$ & 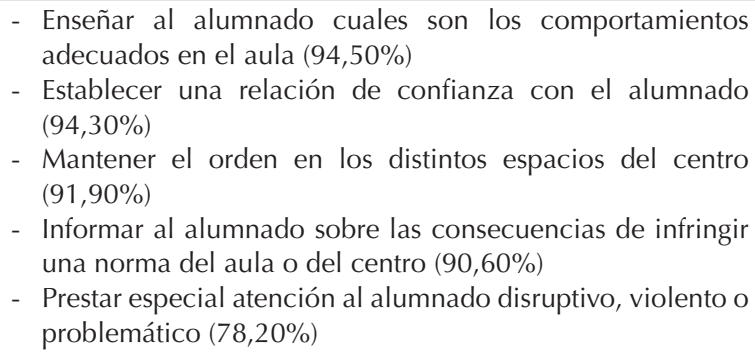 \\
\hline $\begin{array}{l}\text { Estudio Estatal/ } \\
\text { Nacional }\end{array}$ & $\begin{array}{l}\text { Rey y Marchesi } \\
(2005)\end{array}$ & $\begin{array}{l}\text { - Dejar claras las normas de convivencia del centro }(81,40 \%) \\
\text { - Enseñar al alumnado a relacionarse de forma positiva con } \\
\text { otras personas }(73,10 \%) \\
\text { - } \text { Mantener el orden en las aulas }(72,40 \%) \text { y en el centro } \\
(68,10 \%) \\
\text { - Elaborar de forma conjunta entre el profesorado y el } \\
\text { alumnado las normas de convivencia en las aulas }(48,10 \%)\end{array}$ \\
\hline Canarias & Rodríguez (2005) & $\begin{array}{l}\text { - Celebrar asambleas de aula en las tutorías }(52,40 \%) \\
\text { - Organizar charlas colectivas de bullying en las tutorías } \\
(51,30 \%) \\
\text { - Debatir el profesorado y el alumnado sobre la convivencia } \\
\text { y el bullying }(42 \%) \\
\text { - Entrevistar al alumnado de riesgo }(41,60 \%)\end{array}$ \\
\hline Galicia & Jares (2006) & $\begin{array}{l}\text { - Formar al profesorado en dinámicas de análisis y resolución } \\
\text { de conflictos }(90,30 \%)\end{array}$ \\
\hline
\end{tabular}




\begin{tabular}{|c|c|c|}
\hline $\begin{array}{l}\text { Lugar de } \\
\text { realización }\end{array}$ & ESTUDIOS & ACCIONES PREVENCIÓN PRIMARIA Y SECUNDARIA** \\
\hline Madrid & $\begin{array}{l}\text { Marchesi, Martín, } \\
\text { Pérez y Díaz (2006) }\end{array}$ & $\begin{array}{l}\text { - Trabajar en tutorías la convivencia y el respeto mutuo } \\
(84,10 \%) \\
\text { - Desarrollar programas de convivencia en el centro }(45,10 \%) \\
\text { - Sensibilizar al profesorado acerca de las características } \\
\text { personales del alumnado }(33,90 \%)\end{array}$ \\
\hline Andalucía & $\begin{array}{l}\text { Alonso, Lobato, } \\
\text { Hernando y Montilla } \\
\text { (2007) }\end{array}$ & $\begin{array}{l}\text { - Sensibilizar al alumnado de la problemática del bullying (86\%) } \\
\text { - Realizar programas de convivencia }(85 \%) \\
\text { - Favorecer metodologías participativas (58\%) } \\
\text { - Trabajar la convivencia y el bullying en las tutorías (52\%) } \\
\text { - Debatir las normas de convivencia }(40 \%)\end{array}$ \\
\hline $\begin{array}{l}\text { Estudio Estatal/ } \\
\text { Nacional }\end{array}$ & $\begin{array}{l}\text { Del Barrio et al. } \\
\text { (2007) }\end{array}$ & $\begin{array}{l}\text { - Trabajar convivencia y conocimiento mutuo en las tutorías } \\
(95 \%) \\
\text { - Debatir las normas del Reglamento de Régimen Interno - } \\
\text { RRI- del centro }(75 \%) \\
\text { - Incluir en las programaciones académicas actividades sobre } \\
\text { convivencia }(67,60 \%) \\
\text { - Sensibilizar al profesorado sobre las características } \\
\text { - personales del alumnado }(61,50 \%) \\
\text { - Utilizar metodologías participativas }(47,50 \%)\end{array}$ \\
\hline $\begin{array}{l}\text { Comunidad } \\
\text { Valenciana }\end{array}$ & $\begin{array}{l}\text { Marchesi, Pérez, } \\
\text { Pérez y Marín (2007) }\end{array}$ & $\begin{array}{l}\text { - Desarrollar programas de mejora de convivencia }(93,70 \%) \\
\text { - Tratar temas de convivencia en las tutorías }(82,50 \%) \\
\text { - Enseñar al alumnado a relacionarse de forma positiva con } \\
\text { otras personas ( } 84 \%) \\
\text { - Tener en cuenta las opiniones del alumnado para resolver } \\
\text { los problemas }(70,30 \%) \\
\text { - Informar al profesorado sobre las características personales } \\
\text { del alumnado }(36,90 \%)\end{array}$ \\
\hline $\begin{array}{l}\text { Estudio Estatal/ } \\
\text { Nacional }\end{array}$ & $\begin{array}{l}\text { Díaz-Aguado, } \\
\text { Martínez y Martín } \\
\text { (2010) }\end{array}$ & $\begin{array}{l}\text { - Mantener el orden en las clases }(89,50 \%) \\
\text { - Desarrollar actividades para que la clase esté más unida } \\
(64,90 \%) \\
\text { - Aplicar el aprendizaje cooperativo }(64,10 \%) \\
\text { - Reunir periódicamente al profesorado para debatir sobre } \\
\text { cómo mejorar la convivencia }(63,50 \%) \\
\text { - Programar las materias de modo que contribuyan a generar } \\
\text { un adecuado clima de convivencia }(63,50 \%)\end{array}$ \\
\hline Islas Baleares & $\begin{array}{l}\text { Instituto para la } \\
\text { Convivencia y el } \\
\text { Éxito Escolar del } \\
\text { Gobierno Balear } \\
\text { (2011) }\end{array}$ & $\begin{array}{l}\text { - Mantener el orden en la clase }(91,10 \%) \\
\text { - } \text { Adaptar los contenidos para conseguir un buen clima de } \\
\text { convivencia }(88,40 \%) \\
\text { - Desarrollar actividades para que el grupo-clase esté más } \\
\text { - Unido }(68,40 \%) \\
\text { - Utilizar el aprendizaje cooperativo }(65,20 \%) \\
\text { - Estimular al alumnado a discutir sobre cuestiones sociales y } \\
\text { políticas en las que hay diversidad de opiniones }(64,30 \%)\end{array}$ \\
\hline País Vasco & $\begin{array}{l}\text { Arregi y Martínez } \\
\text { (2012) }\end{array}$ & $\begin{array}{l}\text { - Contar con un Reglamento de Régimen Interno }(88,20 \%) \\
\text { - Contar con profesorado preparado y que trabaja } \\
\text { coordinadamente }(84,60 \%) \\
\text { - Aplicar programas de convivencia }(82,20 \%) \\
\text { - Favorecer la participación e implicación del profesorado en } \\
\text { la organización del centro }(70,60 \%)\end{array}$ \\
\hline
\end{tabular}




\begin{tabular}{ccc}
\hline $\begin{array}{c}\text { Lugar de } \\
\text { realización }\end{array}$ & ESTUDIOS & ACCIONES PREVENCIÓN PRIMARIA Y SECUNDARIA** \\
\hline $\begin{array}{c}\text { Valencia } \\
\text { (Provincia) }\end{array}$ & Serrano (2013) & - Promover aceptación y respeto en aula $(70,83 \%)$ \\
& - Promover aceptación y respeto en centro $(80,36 \%)$ \\
- & Marcar las normas del aula y las consecuencias de su \\
& trasgresión $(51,19 \%)$ \\
- & Considerar el bullying en el Plan de Convivencia, en el \\
& Reglamento de Régimen Interno y en el Plan de Acción \\
& Tutorial $(45,83 \%)$ \\
- & Aumentar la vigilancia en el centro (33,93\%) \\
- & Consensuar las normas del aula y las consecuencias de su \\
& transgresión $(30,95 \%)$ \\
- & Fomentar el uso de metodologías de aprendizaje \\
& Cooperativo (30,95\%) \\
- & Consensuar las normas del centro y consecuencias \\
& transgresión $(28,57 \%)$ \\
- & Hablar de bullying con el alumnado (25\%) \\
- & Concienciar de la importancia del bullying (23,81\%)
\end{tabular}

*En bibliografía aparece la referencia del estudio completa

**El \% hace referencia al porcentaje de profesorado que tras ser preguntado en los estudios presentados responde realizar esa acción de prevención primaria y secundaria.

Fuente: Elaboración propia.

En síntesis de estos resultados podemos señalar, al igual que Sag (2008), que para la prevención primaria se desarrollan acciones de carácter general como jornadas de sensibilización dirigidas al alumnado o acciones de tipo curricular como abordar la prevención del acoso desde el Proyecto Educativo de Centro y los Proyectos Curriculares así como acciones relativas a la organización y funcionamiento del centro, donde se incluye el desarrollo de normas de convivencia, aumentar la vigilancia de posibles lugares de riesgo, generar distintas vías anónimas de denuncia como un buzón; o formar al profesorado en bullying, entre otras. López, Domínguez y Álvarez (2010), destacan acciones como los círculos (Smith, y Sharp, 1994), la ayuda entre iguales, la dramatización o role-playing, reuniones entre padres, profesorado y alumnado, programas de formación del profesorado, crear un día de no violencia y paz, utilizar el aprendizaje cooperativo como metodología de trabajo y que el profesorado trabaje coordinadamente con otros/as profesionales del campo de la sanidad, la justicia y los servicios sociales. Además, remarcan que se realicen acciones que impliquen a todos los miembros de la comunidad educativa (profesorado, familias y alumnado), como bien indican Debarbieux y Blaya (2010) y Cerezo, Calvo y Sánchez (2010), dado que de este modo se aborda la problemática del acoso escolar o bullying de forma global y comunitaria.

En relación a las acciones de prevención secundaria, se deduce que las familias y el profesorado deben ser conocedores de posibles factores que pueden promover o inhibir la aparición de situaciones de bullying, pudiendo así tratar de suscitar aquellos factores que protejan a los/las menores de la aparición de posibles casos de acoso escolar e intentar eliminar aquellos que supongan algún riesgo. Factores que inciden en los casos de bullying y que pueden ser como clasifica Avilés (2002) de tipo 
personal, como el temperamento, el sexo o la edad; de tipo escolar, entre los que se podría señalar el tamaño del centro y del aula, la organización y gestión de la disciplina en el centro y la formación en acoso escolar del profesorado; de tipo familiar, como la actitud emotiva de los padres y madres hacia el/la adolescente, los métodos de afirmación de la autoridad empleados y el clima de convivencia familiar; y de tipo social, como la situación de bienestar del entorno en el que vive el/la menor y la constancia en el tiempo de malas relaciones.

\section{Resultados en el nivel de prevención terciaria}

Según los hallazgos, se trata de aquella acción de intervención a ejecutar cuando todas las acciones anteriores han sido en vano y aparece una situación problema, que hay que detener y evitar que vuelva a aparecer a través de acciones correctivas (Garaigordobil y Oñederra, 2010) -ver tabla 2-.

Tabla 2. Acciones de prevención terciaria usadas por profesorado de ESO

\begin{tabular}{|c|c|c|}
\hline $\begin{array}{l}\text { Lugar de } \\
\text { realización }\end{array}$ & ESTUDIOS* & ACCIONES PREVENCIÓN TERCIARIA** \\
\hline Cataluña & Badia (2001) & $\begin{array}{l}\text { - Parar la clase cuando el alumnado se ríe de forma } \\
\text { molesta }(47,60 \%) \text {, da vueltas por la clase }(42,49 \%), \\
\text { interviene sin permiso }(42,20 \%) \text { y se tira objetos } \\
\text { sobre la mesa haciendo ruido }(41,20 \%) \\
\text { - Mirar de forma intimidatoria al alumnado que está } \\
\text { fuera de su asiento (con un } 25,30 \%) \\
\text { - Avisar a los padres cuando el alumnado no asista } \\
\text { a clase ( } 70 \%) \text {, no trabaje }(54,10 \%) \text { o se niegue a } \\
\text { hacer lo que dice el profesor/a }(36,50 \%)\end{array}$ \\
\hline Ceuta & Pareja (2002) & $\begin{array}{l}\text { - Hablar a solas con el alumnado cuando este ignora } \\
\text { a un/a compañero/a }(43,80 \%) \text {, le impide participar } \\
\text { en actividades }(30,80 \%) \text { o habla mal de alguien } \\
(42,90 \%) \\
\text { - Hablar con el grupo-clase cuando alguien Ilama } \\
\text { por motes a algún compañero/a }(28,60 \%)\end{array}$ \\
\hline $\begin{array}{l}\text { Estudio } \\
\text { Estatal/ } \\
\text { Nacional }\end{array}$ & $\begin{array}{l}\text { Rey y Marchesi } \\
(2005)\end{array}$ & $\begin{array}{l}\text { - Resolver el problema teniendo en cuenta las opiniones } \\
\text { del profesorado, alumnado y familias }(94 \%) \\
\text { - Dialogar para llegar a un acuerdo }(90,60 \%) \\
\text { - Aplicar castigos y sanciones }(82 \%)\end{array}$ \\
\hline Canarias & Rodríguez (2005) & - Seguir un proceso de mediación $(44,60 \%)$ \\
\hline Aragón & $\begin{array}{l}\text { Gómez-Bahillo et } \\
\text { al. (2006) }\end{array}$ & $\begin{array}{l}\text { - Hablar con al acosador/a y hacerle reflexionar } \\
\text { sobre su actitud }(44,30 \%) \\
\text { - Plantear el caso en clase para tomar medidas entre } \\
\text { todos/as }(2,60 \%)\end{array}$ \\
\hline Cantabria & González (2006) & $\begin{array}{l}\text { - Hablar con los acosadores/as y con las víctimas } \\
(24 \%)\end{array}$ \\
\hline
\end{tabular}




\begin{tabular}{cll}
\hline $\begin{array}{c}\text { Lugar de } \\
\text { realización }\end{array}$ & \multicolumn{1}{c}{ ESTUDIOS* } & \multicolumn{1}{c}{ ACCIONES PREVENCIÓN TERCIARIA** } \\
\hline Andalucía & $\begin{array}{l}\text { Alonso, Lobato, } \\
\text { Hernando y }\end{array}$ & $\begin{array}{l}\text { - Hablar con los implicados/as }(90 \%) \\
\text { - Tratar el problema en las tutorías (80\%) }\end{array}$ \\
& Montilla (2007) & $\begin{array}{l}\text { - Hablar del caso de bullying con las familias (79\%) } \\
- \text { Derivar al alumnado al orientador/a (55\%) }\end{array}$ \\
\hline
\end{tabular}

\begin{tabular}{ll}
\hline Estudio & Del Barrio et al. \\
Estatal/ & $(2007)$ \\
Nacional &
\end{tabular}

- Hablar a solas con el alumnado cuando ignora a un compañero/a (81\%), hablan mal de él/ella $(77,40 \%)$, le ponen motes $(71,40 \%)$, le insultan $(68,90 \%)$, le obligan con amenazas a hacer algo que no quiere $(67,10 \%)$ y le amenazan para meterle miedo $(64 \%)$

- Hablar sobre el tema en clase si no dejan participar a alguien $(68,20 \%)$, le esconden cosas $(70,40 \%)$ y se las rompen $(61,80 \%)$

Comunidad Marchesi, Pérez, - Hablar con el alumnado implicado cuando se Valenciana Pérez y Marín impide participar a un compañero/a en alguna (2007) actividad $(90,50 \%)$, se le ignora $(90,40 \%)$, se habla mal de alguien $(86,10 \%)$, se le ponen motes $(73,80 \%)$, si se insulta $(65,80 \%)$ y si se rompe algún material $(47,10 \%)$

\begin{tabular}{|c|c|c|}
\hline $\begin{array}{l}\text { Estudio } \\
\text { Estatal/ } \\
\text { Nacional }\end{array}$ & $\begin{array}{l}\text { Díaz-Aguado, } \\
\text { Martínez y Martín } \\
(2010)\end{array}$ & $\begin{array}{l}\text { - Discutir los problemas de convivencia y disciplina } \\
\text { con el alumnado }(79,20 \%)\end{array}$ \\
\hline $\begin{array}{l}\text { Islas } \\
\text { Baleares }\end{array}$ & $\begin{array}{l}\text { Instituto para la } \\
\text { Convivencia y el } \\
\text { Éxito Escolar del } \\
\text { Gobierno Balear } \\
(2011)\end{array}$ & $\begin{array}{l}\text { - Discutir los problemas de convivencia con el } \\
\text { alumnado }(84,80 \%)\end{array}$ \\
\hline Valencia & Serrano (2013) & $\begin{array}{l}\text { - Aplicar las acciones del Plan de Convivencia, del } \\
\text { Reglamento de Régimen Interno y del Plan de } \\
\text { Acción Tutorial establecidas }(89,29 \%) \\
\text { - Hablar en privado con la víctima }(83,33 \%) \\
\text { - Derivar al departamento de orientación al acosador/a } \\
\text { (54,76\%) y a la víctima (70,83\%) } \\
\text { - Aplicar el Reglamento de Régimen Interno y las } \\
\text { - normas de clase al acosador }(70,83 \%) \\
\text { - Derivar al acosador/a al equipo directivo (54,17\%) } \\
\text { - Colaborar con acciones propuestas por los Servicios } \\
\text { - Sociales y Consejería de educación }(48,81 \%) \\
\text { - Hablar con el alumnado (45,83\%) } \\
\text { - Colaborar con el departamento de orientación del } \\
\text { centro (39,88\%) } \\
\text { - Aplicar las normas de clase }(35,71 \%) \\
\text { - Hablar con las familias }(20,24 \%)\end{array}$ \\
\hline
\end{tabular}

*En bibliografía aparece la referencia del estudio completa

**El \% hace referencia al porcentaje de profesorado que tras ser preguntado en los estudios presentados responde realizar esa acción de prevención terciaria.

Fuente: Elaboración propia. 
En síntesis, señalar que las estrategias a utilizar en este nivel son las asambleas de aula o el Método Pikas (Pikas, 1989) y van desde las de carácter educativo a las punitivas o sancionadoras. Según Sugai y Horner (2002), hasta los años 60, las acciones más frecuentes fueron de carácter punitivo y consistían en actos de maltrato físico como bofetadas o golpes con la regla, prohibidos en España desde que se aprobó la Ley Orgánica 8/1995 reguladora del Derecho a la Educación (LODE). Sin embargo, algunos estudios de revisión como el realizado Martínez-Otero (2005) todavía muestran como las prácticas de castigos basados en las amenazas y el miedo, siguen al uso. Para nosotras, tal y como señalaba Skinner (1975), los castigos sirven para detener la manifestación de conductas negativas en un momento dado. Sin embargo, tienen como inconveniente el no evitar que dichas conductas puedan manifestarse en un futuro inmediato tras la retirada del estímulo punitivo. Además, los castigos pueden traer consigo otros efectos negativos, como por ejemplo, que los niños/as y jóvenes que han recibido sanciones demasiado severas y desagradables reproduzcan este comportamiento en su etapa adulta con otros seres más débiles (Walters y Grusec, 1977) o incluso pueden desatar en el menor un sentimiento de frustración y rencor que le lleve a comportarse en el fututo de forma agresiva (Azorin y Holz, 1966).

Investigadores como Stainton y Willig (2008) indican que el profesorado ha de evitar ser demasiado autoritario en el manejo del poder y control sobre el alumnado, debiendo adoptar actitudes más conciliadoras y consensuadas basadas en el uso del diálogo como estrategia educativa. Asimismo, Barri (2006), Martínez-Otero (2005) y Orte (2003), aseguran que ante una situación de acoso escolar se deben seguir, siempre que sea posible, acciones educativas orientadas a reparar el daño causado por el acosador/a y a enseñar al alumnado qué debe hacer. Además, señalan que es necesario realizar un estudio previo del acosador/a que lleve a averiguar la causalidad de su comportamiento, compensando posibles carencias afectivas, relacionales... que hayan propiciado la situación problema. López, Domínguez y Álvarez (2010), proponen el entrenamiento de la asertividad; el desarrollo de la empatía; aplicar el proceso de mediación (Torrego 2000); el Método Pikas (Pikas, 1989); el Modelo de intervención de Lera y Cela (2000); el Modelo integrado de mejora de la convivencia (Torrego, 2006); el Programa Concienciar, Informar y Prevenir (Cerezo, Calvo y Sánchez, 2010) y establecer un aula de convivencia.

Hemos hallado estudios que defienden los castigos, como técnica educativa, (Otero, 2005) alegando que son eficaces para eliminar rápidamente conductas inadecuadas, aunque hay que tener en cuenta que a menudo lo que se consigue es que el/la jóven oculte el comportamiento disruptivo de la mirada adulta pero no la desaparición de las conductas no adecuadas -ver Tabla 3-.

Se observa en estos resultados que el profesorado ante casos de acoso escolar menos graves utiliza, acciones educativas basadas fundamentalmente en el diálogo aunque a medida que aumenta la peligrosidad de los hechos, crece también el uso de acciones punitivas como partes, sanciones y expedientes. De hecho, estudios como el de Badia (2001), Del Barrio et al. (2007), Marchesi, Pérez, Pérez y Marín (2007) y Pareja (2002) indican que el profesorado emplea acciones de prevención terciaria punitivas ante el alumnado con comportamientos disruptivos -ver tabla 2-, como la separación del acosador/a de su víctima y del resto de sus compañeros/as del grupoclase. Sin embargo, según Badia (2001) sería una buena opción combinar ambos 
tipos de acciones de prevención terciaria, punitivas y reeducadoras, porque así el acosador/a aprende qué no hacer y también qué hacer. De esta manera, el castigo de la conducta disruptiva siempre ha de ir acompañado de la enseñanza de conductas adecuadas dado que, en caso contrario se centra toda la atención en el comportamiento negativo y no se le muestra al alumnado lo que se espera de él/ella, pudiendo causarle cierta confusión.

Tabla 3. Acciones de prevención terciaria punitivas y sancionadoras usadas por profesorado de ESO

\begin{tabular}{|c|c|c|}
\hline $\begin{array}{l}\text { Lugar de } \\
\text { realización }\end{array}$ & ESTUDIOS* & $\begin{array}{l}\text { ACCIONES DE PREVENCIÓN TERCIARIA } \\
\text { PUNITIVAS Y SANCIONADORAS** }\end{array}$ \\
\hline Nacional & $\begin{array}{l}\text { Serrano y Iborra } \\
(2005)\end{array}$ & $\begin{array}{l}\text { - Imponer una sanción al acosador/a }(36,10 \%) \\
\text { - Abrir un expediente }(18 \%) \\
\text { - Expulsar de clase al alumnado }(6,60 \%) \\
\text { - Expulsar del centro educativo al alumnado durante } \\
\text { varios días }(6,60 \%)\end{array}$ \\
\hline Alicante & Caruana (2005) & $\begin{array}{l}\text { - Expulsar, separar o aislar al alumnado conflictivo del } \\
\text { resto de sus compañeros/as }(13,22 \%)\end{array}$ \\
\hline Galicia & Jares (2006) & $\begin{array}{l}\text { - Sacar al alumnado con comportamientos disruptivos de } \\
\text { las clases para poder desarrollar estas con normalidad } \\
(71,80 \%)\end{array}$ \\
\hline Valencia & Serrano (2013) & - Derivar casos a la Fiscalía de Menores (23,81\%) \\
\hline
\end{tabular}

Fuente: Elaboración propia.

\section{CONCLUSIÓN Y DISCUSIÓN}

Atendiendo al objetivo de este estudio, indagar sobre el tipo de acciones que el profesorado utiliza ante el acoso escolar en los Institutos de ESO en España, los resultados obtenidos en esta investigación permiten afirmar que las acciones de prevención primaria y secundaria que más utiliza el profesorado de ESO en las aulas y centros educativos del contexto educativo español son las relacionadas con establecer un sistema de normas de convivencia, tanto a nivel de centro como en cada una de las aulas, puesto que ayuda a mantener cierto orden; trabajar contenidos sobre convivencia y acoso escolar en las tutorías y en las distintas áreas curriculares además de tratar de unir al alumnado que conforma un mismo grupo-clase, siendo de ayuda el empleo de metodologías cooperativas y colaborativas. Se trata de acciones con un enfoque más educativo que punitivo y/o sancionador. Resaltar, no obstante, que entre estas acciones más comunes hay una omisión casi completa de acciones en las que aparezca el binomio profesorado-alumnado. Del mismo modo, la utilización de acciones a nivel de centro y con las familias son muy escasas. Desde este estudio sin embargo, se plantea la necesidad de considerar como imprescindible 
la participación de todas las personas implicadas para generar una cultura escolar que actúe en el beneficio común de la sociedad y de los estudiantes en particular.

Destacar también, a partir de los resultados obtenidos, que a nivel de prevención terciaria los estudios resaltan que las acciones más utilizadas por el profesorado en los grupos-clase son: hablar con el alumnado implicado, aplicar las normas, derivar el caso al departamento de orientación, así como las marcadas por su estilo educativo y personal (Vidal, 2000).

Somos conscientes que en España desde que se implanta la Ley Orgánica de Ordenación General del Sistema Educativo (LOGSE) de 1990, aparece un gran interés por el tratamiento pedagógico de los aspectos educativos que trabajan problemas sociales, que ya no deja de estar presente en ninguna de las leyes posteriores. Al revisar, la Ley de Educación (LOE) de 2006, y concretamente su declaración de fines y principios, se observa la necesidad de guiar la educación de las personas y las sociedades hacia los valores de tolerancia, solidaridad, paz y libertad así como hacia el pluralismo, la participación y el respeto.

Actualmente, en la Ley Orgánica para la Mejora de la Calidad Educativa (LOMCE) de 2013, se resalta el trabajo que debe hacerse en los centros educativos para la inclusión de todos y todas a través del fomento de actitudes que no acepten el acoso escolar. Como señala Pastor (2015) los valores en el currículo han de basarse en fomentar valores de tipo social, a través de un aprendizaje de la prevención y la resolución pacífica de conflictos en todos los ámbitos de la vida (personal, familiar y social).

Siguiendo las conclusiones de estudios como el de Hernández y Sancho (2004) y el del Instituto para la Convivencia y el Éxito Escolar del Gobierno Balear (2011), es necesario mejorar la comunicación y coordinación entre los diferentes miembros de la comunidad educativa; que todos los centros educativos cuenten con un equipo de mediación así como fomentar actividades extraescolares y complementarias que ayuden a unir al alumnado y creen un buen clima de convivencia. Además, es necesario dotar a los centros de los recursos humanos y materiales adecuados que permitan disminuir la masificación en las aulas e incidir así en la consecución de una mayor calidad educativa. También como destacan Alonso (2009), Jares (2006), Nicolaides, Toda y Smith (2002), Rodríguez (2005) y Yoon (2004), es relevante, para abordar adecuadamente estos casos en los centros y aulas escolares, seguir adquiriendo formación que permita potenciar la cultura global y de comunidad educativa de los Institutos de ESO para saber gestionar el acoso escolar que involucre a la víctima, al acosador/a, sus familias y al grupo-clase.

Según Echeita (2012), la enseñanza es una actividad de aprendizaje constante por lo tanto el profesorado, el equipo directivo y los integrantes del departamento de orientación tienen la responsabilidad de formarse durante toda su vida profesional, velando por la convivencia escolar (Serrano, 2013). Así mismo, hemos de ser conscientes de las nuevas formas de comunicarse y de relacionarse que tienen nuestros estudiantes a través de las redes sociales y sus aplicaciones. El Instituto Nacional de estadística, en su informe de 2014, muestra la elevada proporción de uso de las tecnologías de la información entre la población de menores y adolescentes: el 92\% de menores entre los 10 y los 15 años son usuarios/as de Internet. Bajo el entorno 
de dichas redes, están apareciendo nuevas formas de acoso y maltrato como es el cyberbullying que incluye según Kowalski, Limber y Agatston (2010) y Buelga, Cava y Musitu (2010), cualquier forma de agresión o acoso ejercida a través del envío de correos electrónicos, mensajerías instantáneas, mensajes de móvil, por medio de páginas web, blogs, chats, coloquios on-line y otras TICs.

Así pues, y siguiendo a Valverde (2013), sabemos que los programas anti-bullying que han reducido la prevalencia del acoso escolar han sido aquellos en los que existe una implicación continuada del profesorado y se han complementado con su formación sobre bullying y ciberbullying para adecuar y ajustar sus intervenciones (Gabarda, 2015). Además, y dado que las leyes educativas buscan la inclusión -la creación de un marco escolar respetuoso con la persona (Callado et al., 2015)- para brindar oportunidades efectivas de aprendizaje y participación a todo el alumnado (Naula y Calderón, 2015), debemos realizar un proceso sistémico para aplicar adecuados valores personales y sociales a la acción educativa (Booth, 2006), planteando acciones de prevención e intervención educativa con toda la comunidad (alumnado, profesorado, familia, centro y sociedad) para avanzar en la erradicación de este fenómeno en los centros educativos.

\section{REFERENCIAS BIBLIOGRÁFICAS}

Alonso, P. (2009). El acoso escolar: análisis desde la perspectiva de profesores en formación y profesores en activo. Revista de Pedagogía Bordón, 61(3), 7-18.

Alonso, P., Lobato, H., Hernando, Á. y Montilla, Mª V. C. (2007). La visión del acoso escolar en futuros profesores de primaria y de secundaria. En J. J. Gázquez, $M^{a}$. C. Pérez., A. J. Cangas y N. Yuste (Eds.), Mejora de la convivencia y programas encaminados a la prevención e intervención del acoso escolar (pp. 105-110). Granada: Grupo Editorial Universitario.

Arregi, A. y Martínez, P. (2012). El maltrato entre iguales en educación primaria y ESO. País Vasco: Instituto Vasco de Evaluación e Investigación Educativa.

Avilés, J. Ma . (2002). La intimidación y el maltrato entre iguales (bullying) en la educación secundaria obligatoria. Validación del cuestionario CIMEI y estudio de incidencia (Tesis Doctoral). Universidad de Valladolid, Facultad de Psicología.

Azorin, N. H. y Holz, W. C. (1966). Punishment. En W. K. Honing (Ed.), Operant Behavior: áreas of research and application. Nueva York: Appleton-Century-Crofts.

Badia, Ma. M. (2001). Las percepciones de profesores y alumnos de ESO sobre la intervención en el comportamiento disruptivo: un estudio comparativo de los IES y escuelas de enseñanza secundaria de la comarca del Bages (Tesis Doctoral). Universidad Autónoma de Barcelona, Departamento de Psicología de la Educación.

Barri, F. (2006). SOS bullying: prevenir el acoso escolar y mejorar la convivencia. Madrid: Wolters Kluwer España / Educación.

Bisquerra, R. y Martínez, M. (1998). El clima escolar als centres d'ensenyament secundari a Catalunya. Barcelona: Generalitat de Catalunya, Departament d'Ensenyament, Consell Superior d'Avaluació del Sistema Educatiu.

Booth, T. (2006). Manteniendo el futuro con vida; convirtiendo los valores de la inclusión en acciones. En M. A. Verdugo y F. B. Jordán de Urríes (Coords.), 
Rompiendo inercias. Claves para avanzar. Comunicación presentada a las VI Jornadas Científicas de Investigación sobre Personas con Discapacidad (pp. 211 217). Salamanca: Amarú.

Buelga, S., Cava. M. J. y Musitu, G. (2010). Cyberbullying: victimización entre adolescentes a través del teléfono móvil y de Internet. Psicothema, 22, 784-789.

Callado, J. A., Molina, Mª D., Pérez, E. y Rodríguez, J. (2015). La educación inclusiva en los colegios de zonas rurales. New approaches in educational research, 2(4), 115-123. DOI: http://doi.org/ 10.7821/naer.2015.4.120.

Caruana, A. (Coord.). (2005). Programa de Educación Emocional para la Prevención de la Violencia, $2^{\circ}$ ciclo de ESO. Valencia: Consejería de Cultura, Educación i Deporte. Recuperado de http://213.0.8.18/portal/Educantabria/RECURSOS/Materiales /Biblinter/Valencia_Programa_Educa_emocional_2_CicloESO.pdf.

Cerezo, F. (2012). Psique: Bullying a través de las TIC. Boletín Científico Sapiens Research, 2(2), 24-29.

Cerezo, F., Calvo, Á. R. y Sánchez, C. (2010). Programa CIP. Intervención psicoeducativa y tratamiento diferenciado del bullying, Concienciar, Informar Prevenir. Madrid: Pirámide.

Chaux, E. (2012). Educación, convivencia y agresión escolar. Bogotá: Editorial Taurus.

Debarbieux, E. y Blaya, C. (2010). Sociología y violencia escolar: un enfoque contextual. En R. Ortega (Ed.), Agresividad injustificada, Bullying y violencia escolar. Madrid: Alianza.

Defensor del Pueblo (2007). Violencia Escolar: El Maltrato entre Iguales en la Educación Secundaria Obligatoria 1999-2006. Comité Español de UNICEF Madrid: Publicaciones de la Oficina del Defensor del Pueblo. Recuperado de http://www. defensordelpueblo.es/es/Documentacion/Publicaciones/monografico/contenido_1261583505460.html.

Del Barrio, C., Espinosa, M. A., Martín, E., Ochaita, E., Barrios, A, De Dios, M., ... y UNICEF. (2007). Violencia escolar: El maltrato entre iguales en la Educación Secundaria Obligatoria 1999-2006. Madrid: Publicaciones de la oficina del Defensor del Pueblo. Recuperado de http://www.defensordelpueblo.es/es/Documentacion/ Publicaciones/monografico/Documentacion/Informe_violencia_escolar_ESO.pdf.

Del Barrio, C. (2013). Experiencias de acoso y ciberacoso: autores, autoras, víctimas y consideraciones para la prevención. Convives, 3, 25-33. Recuperado de http:// convivenciaenlaescuela.es/?p=463.

Díaz-Aguado, Ma. J., Martínez, R. y Martín, J. (2010). Estudio Estatal sobre la Convivencia Escolar en la Educación Secundaria Obligatoria. Madrid: Ministerio de Educación. Recuperado de https://sede.educacion.gob.es/publiventa/ detalle. action?cod=13567.

Echeita, G. (2011). El proceso de inclusión educativa en España. ¡Quien bien te quiere te hará Ilorar! Participación Educativa, 18, 117-128.

Garaigordobil, M. (2011). Prevalencia y consecuencias del cyberbullying: una revisión. International Journal of Psychology and Psychological Therapy, 11(2), 233254. Recuperado de http://www.redalyc.org/pdf/560/56019292003.pdf. 
Garaigordobil, M. y Oñederra, J. A. (2010). Los centros educativos ante el acoso escolar: actuaciones del profesorado, acciones sancionadoras y actividades de prevención. Información psicológica, 99, 4-18.

Gabarda, S. (2015). Ciberbullying y otras problemáticas en la red en Educación Secundaria Obligatoria. (Trabajo Final de Grado de Educación Social). Universitat de València.

García-Fernández, C. M. (2013). Acoso y ciberacoso en escolares de primaria: factores de personalidad y de contexto entre iguales. (Tesis Doctoral). Universidad de Córdoba. Recuperado de http://helvia.uco.es/xmlui/handle/10396/10949.

Instituto para la Convivencia y el Éxito Escolar. (2011). Estudio sobre la Convivencia Escolar en la Educación Secundaria Obligatoria de la Islas Baleares. Islas Baleares: Consejería de Educación, Cultura y Universidades. Gobierno de las Islas Baleares. Recuperado de http://www.caib.es/sacmicrofront/archivopub.do? ctrl=MCRST15 1 ZI114107\&id=114107.

Instituto Vasco de Investigación e Innovación Educativa. (ISEI-IVEI). (2009). El maltrato entre iguales en Euskadi. Recuperado de http://www.iseiivei.net/cast/pub/ bullying2009/Bullying2009.pdf.

Jäger, T., Amado, J., Matos, A. y Pessoa, T. (2010). Analysis of experts' and trainers' viwer of cyberbullying. Australian Journal of Guidance and Counseling, 20(2), 169-181. DOI: http://doi.org/10.1375/ajgc.20.2.169.

Jares, X. (2006). Conflicto y convivencia en los centros educativos de secundaria. Revista de educación, 339, 467-491. Recuperado de http://www.revistaeducacion. mec.es/re339/re339a21.pdf.

Kowalski, R. M., Limber, S. P. y Agaston, P. (2008). Cyberbullying. Malden, MA: Blackwell Publishing.

Kowalski, R., Limber, S. y Agatston, P. (2010). Cyberbullying: El acoso digital en la era digital. Bilbao: Desclée de Brower.

Lera, M. J. y Cela, D. (2000). ¿Conoces a alguien como Adolfo? Sevilla: Materiales del programa europeo NOVAS-RES.

Ley Orgánica 8/1985, de 3 de julio, reguladora del Derecho a la Educación (LODE). Boletín Oficial del Estado, núm. 159 de 4 de julio de 1985.

Ley Orgánica 1/1990, de 3 de Octubre, de Ordenación General del Sistema Educativo (LOGSE). Boletín Oficial del Estado, de 4 de octubre de 1990.

Ley Orgánica 2/2006, de 3 de Mayo, de Educación (LOE). Boletín Oficial del Estado de 4 de mayo de 2006.

Ley Orgánica 8/2013, de 9 de diciembre, para la Mejora de la Calidad Educativa (LOMCE). Boletín Oficial del Estado, de 10 de diciembre de 2013.

López, A., Domínguez, J. y Álvarez, E. (2010). Bullying vertical: variables predictivas de la violencia escolar. Revista de Investigación en Educación, 8, 24-38.

Marchesi, A., Martín, E., Pérez, E. Ma . y Díaz, T. (2006). Convivencia, conflictos y educación en los centros escolares de la Comunidad de Madrid. Madrid: Defensor del Menor de la Comunidad de Madrid. Recuperado de http://asociacionplazadelcastillo.org/Textosweb/informe_madrid_menores_ 2006/primera_parte_introduccion.pdf. 
Marchesi A., Pérez, F., Pérez, E. y Martín, E. (2007). Un estudio epidemiológico del acoso escolar en centros de Educación Primaria y Secundaria de la Comunidad Autónoma de Valencia. Valencia: Síndic de Greuges de la Comunitat Valenciana.

Martínez-Otero, V. (2005). Conflictividad escolar y fomento de la convivencia. Revista Iberoamericana de Educación, 38, mayo-agosto. Recuperado de http://www. rieoei.org/rie38a02.htm.

Muñoz, J. Mª (2009). Prevención del acoso escolar (bullying). Revista Digital Innovación y Experiencias Educativas, 21, agosto. Recuperado de http://www.csi-csif. es/andalucia/modules/mod_ense/revista/pdf/Numero_21/JOSE\%20MARIA_MUNOZ_VIDAL01.pdf.

Naula, V. F. y Calderón, P. V. (2015). Actitudes de los pares hacia estudiantes de educación inclusiva. Recuperado de http://dspace.ucuenca.edu.ec/bitstream/123456789/21674/1/TESIS.pdf.

Nicolaides, S., Toda, Y. y Smith, P. K. (2002). Kowledge andattitudes about school bullying in trainee teachers. British Journal of Educational Psychology, 72, 105-118.

Orte, C. (2003). Los problemas de convivencia en las aulas. Análisis del bullying. Revista Electrónica Interuniversitaria de Formación del Profesorado, 6(2). Recuperado de http://aufop.com/aufop/uploaded_files/articulos/1227720566.pdf.

Ortega-Ruiz, R., Del Rey R. y Casas, J. A. (2013). La Convivencia Escolar: clave en la predicción del Bullying. Revista Iberoamericana de Evaluación Educativa, 6(2), 91-102.

Ortega-Ruiz, R. (Coord.) (2010). Agresividad injustificada, bullying y violencia escolar. Madrid: Alianza Editorial.

Ovejero, A., Smith, P. K. y Yubero, S. (2013). El acoso escolar y su prevención: perspectivas internacionales. Madrid: Biblioteca Nueva.

Pareja, J. A. (2002). La violencia escolar en contextos interculturales: un estudio en la ciudad autónoma de Ceuta (Tesis Doctoral). Universidad de Granada. Recuperado de http://ldei.ugr.es/cddi/ uploads/tesis/ParejaFernandezReguera2002.pdf.

Pastor, L. (2015). Inclusividad y valores en educación. (Tesis Doctoral). Universidad Complutense de Madrid.

Pikas, A. (1989). The common concer method for the theatment of mobbing. En E. Roland, y E. Munthe (Eds.), Bullying: an international perspective. London: David Fulton.

Rey, R. y Marchesi, Á. (2005). La opinión de los profesores sobre la convivencia en los centros. Madrid: Centro de Innovación Educativa (CIE) e Instituto de Evaluación y Asesoramiento educativo (IDEA). Recuperado de http://213.0.8.18/ portal/ Educantabria/RECURSOS/Materiales/Biblestinv/CIEFUHEM_Encuesta_a_ los_docentes_convivencia_centros-feb_2005.pdf.

Rodríguez, X. (2005). La convivencia en los centros educativos de secundaria de la Comunidad Autónoma Canaria. Islas Canarias: Consejería de Educación, Cultura y Deportes del Gobierno de Canarias. Recuperado de http://213.0.8.18/portal/ Educantabria/RECURSOS/Materiales/Biblestinv/Canarias_Convivencia_ESO.pdf.

Sag, L. (2008). Estrategias de intervención contra la violencia escolar. Revista Digital Innovación y Experiencias educativas, 13, diciembre. Recuperado de http://www. csi-csif.es/andalucia/modules/mod_ense/revista/pdf/Numero_13/LYDIA_SAG_.pdf. 
Serrano, Á. y Iborra, I. (2005). Violencia entre compañeros en la escuela. Valencia: Centro Reina Sofía. Recuperado de http://convivencia.files. wordpress. com/2012/05/informe_reina_sofia-violencia200594p.pdf.

Serrano, M. (2013). Profesorado de Educación Secundaria Obligatoria y Bullying: Prevención e Intervención Educativa. (Tesis Doctoral). Universidad de Valencia, Facultad de Filosofía y Ciencias de la Educación.

Serrate-Mayoral, R. (2007). Bullying, acoso escolar. Guía para entender y prevenir el fenómeno de la violencia en las aulas. Madrid: Laberinto.

Skinner, B. F. (1975). Registro acumulativo. Barcelona: Fontanella.

Smith, P. K. y Sharp, S. (1994). School Bullying. London: Routlege.

Stainton, W. y Willig, C. (2008). The SAGE handbook of qualitative research in psychology. London: Sage Publications.

Sugai, G. y Horner, R. H. (2002). The evolution of discipline practices: School-wide positive behavior supports. Child and Family Behavior Therapy, 24, 23-50.

Torrego, J. C. (2000). Mediación de conflictos en instituciones educativas. Madrid: Narcea.

Torrego, J. C. (2006). Modelo integrado de mejora de la convivencia. Barcelona: Graó.

Valverde, M. A. (2013). Actuaciones para sensibilizar y prevenir el acoso escolar en los centros. Convives, 3, 64-73. Recuperado de http://convivenciaenlaescuela. $\mathrm{es} / \mathrm{p}=463$.

Vidal, M. (2000). Disciplina escolar: Gestión y Control del aula. Madrid: Instituto Calasanz de Ciencias de la Educación.

Walters, G. C. y Grusec, J. E. (1977). Punishment. San Francisco: W. H. Freeman.

Yoon, J. S. (2004). Predicting teacher interventions in bullying situations. Education and Treatment of Children, 27(1), 37-45. 